\title{
Combination of the ratio between metastatic and harvested lymph nodes and negative lymph node count as a prognostic indicator in advanced gastric cancer: a retrospective cohort study
}

\author{
Jianlong Jiang ${ }^{1}$, Jingyao Chen ${ }^{1}$, Hao Zhang ${ }^{2}$, Xionghui Rao ${ }^{3}$, Tengfei Hao ${ }^{1}$, Mingzhe Li ${ }^{1}$, Changhua Zhang ${ }^{1}$, \\ Wenhui $\mathrm{Wu}^{1}$, Yulong $\mathrm{He}^{1}$ \\ ${ }^{1}$ Digestive Diseases Center, Seventh Affiliated Hospital, Sun Yat-sen University, Shenzhen, China; ${ }^{2}$ General Surgery Department, Seventh Affiliated \\ Hospital, Sun Yat-sen University, Shenzhen, China; ${ }^{3}$ Department of Gastrointestinal Surgery, Eighth Affiliated Hospital, Sun Yat-sen University, \\ Shenzhen, China \\ Contributions: (I) Conception and design: C Zhang, Y He; (II) Administrative support: M Li, W Wu; (III) Provision of study materials or patients: \\ C Zhang, Y He; (IV) Collection and assembly of data: J Jiang, J Chen, H Zhang; (V) Data analysis and interpretation: J Jiang, X Rao, T Hao; (VI) \\ Manuscript writing: All authors; (VII) Final approval of manuscript: All authors. \\ Correspondence to: Wenhui Wu; Yulong He. Digestive Diseases Center, Seventh Affiliated Hospital, Sun Yat-sen University, No. 628 Zhenyuan Road, \\ Shenzhen 518107, China. Email: doctorwusysu@126.com; heyulong@mail.sysu.edu.cn.
}

Background: The aim of our study was to examine the impact of the combination of the ratio between metastatic and harvested lymph nodes (RML) and negative lymph node (NLN) count on overall survival (OS) in patients with advanced gastric cancer (GC).

Methods: The clinicopathological data of 2,952 advanced GC patients who received curative resection between 1994 and 2015 were collected. They were divided into four groups according to the RML: 0, 0-0.1, $0.1-0.4$, and $>0.4$. We distinguished survival differences through Kaplan-Meier analysis among the subgroups to investigate the impacts of the RML on OS in advanced GC patients. OS was examined according to clinicopathological variables. Spearman's correlation coefficient was used to assess the relationships between the RML and metastatic lymph node (MLN) count and NLN count.

Results: A total of 1,182 patients were enrolled into the study. The median follow-up time was 39 months (interquartile range 20 to 68 months). The 5-year OS rate of all 1,182 GC patients was 54.4\%. Kaplan-Meier survival analysis showed that the median OS declined significantly with increasing RML (5-year survival rate $81.2 \%$ vs. $69.1 \%$ vs. $42.8 \%$ vs. $13.1 \%, \mathrm{P}<0.001)$. As the NLN count increased, the survival rate of GC patients increased (5-year survival rate $12.8 \%$ vs. $25.2 \%$ vs. $60.2 \%, \mathrm{P}<0.05$ ). The RML, not NLN count, was identified as an independent factor for OS $(\mathrm{P}<0.001)$ through multivariate analysis. Spearman correlation analysis suggested that the RML was positively correlated with the number of MLNs $(\rho=0.973, P<0.001)$ and inversely associated with the NLN count $(\rho=-0.513, \mathrm{P}<0.001)$.

Conclusions: The RML is an independent prognostic predictor of OS in advanced GC patients, and the NLN count may serve as a supplementary strategy for the present tumor-node-metastasis (TNM) classification to further improve the prognostic prediction efficiency. The combination of the RML and NLN count should be an important predictor for current clinical applications.

Keywords: Gastric cancer (GC); prognosis; negative lymph node (NLN); metastatic lymph node (MLN)

Submitted Apr 15, 2021. Accepted for publication Aug 05, 2021.

doi: 10.21037/jgo-21-212

View this article at: https://dx.doi.org/10.21037/jgo-21-212 


\section{Introduction}

Gastric cancer (GC) is the fourth most common cancer in men and the fifth most common in women worldwide (1). It is also the second most common cause of death from cancer (2). Lymph node metastasis is the main metastatic mode of GC and an important cause of postoperative recurrence and death. The most important predictive factor of GC patient prognosis is lymph node status. More than half of GC patients are initially diagnosed with lymph node metastasis (3). Accurate postoperative staging and prognostic evaluation depend on a precise evaluation of lymph node status. Postoperative pathological lymph node (pN) staging should consider the number of metastatic lymph nodes (MLNs) rather than their anatomical site according to the American Joint Committee on Cancer (AJCC) (4). However, whether the current $\mathrm{pN}$ staging is the appropriate strategy for the assessment of overall survival (OS) is still controversial. Many researchers have reported that an insufficient number of harvested lymph nodes (HLNs) can induce stage migration (5). The current TNM staging strategy is dependent only on the number of MLNs; hence, it has an insufficient effect when the HLN count is not enough. Thus, we aimed to identify a sensible prognostic marker that does not rely on the range of lymphadenectomy (6). Previous studies have reported that the ratio between metastasis and harvested lymph nodes (RML) is a strong prognostic factor for advanced GC after radical gastrectomy (7-9). Considering that the HLNs of GC are comprised of metastases and negative lymph nodes (NLNs), both are removed from the specimen simultaneously. In theory, the RML should be related to the NLN count, and the NLN count can also serve as a prognostic indicator. However, to the best of our knowledge, no scholars have reported the potential relationship between the RML and NLN count.

Taking into account the above issues, we aimed to elucidate the potential influence of both the RML and NLN count on the prognosis of advanced GC and to determine whether the NLN count could increase the predictive effect of the RML on survival.

We present the following article in accordance with the STROBE reporting checklist (available at https://dx.doi. org/10.21037/jgo-21-212).

\section{Methods}

Patients who underwent curative resection for advanced
GC from January 1994 to December 2015 were identified from the GC database of the First Affiliated Hospital of Sun Yat-sen University (FAHSYSU) in Guangzhou, China. The following patients were excluded: personal history of malignancy, remnant stomach cancer or recurrent carcinoma, preoperative chemotherapy, stage IV and distant metastasis (e.g., lung, liver, brain, or bone marrow metastasis) and early GC, non-curative resection, $<15$ HLNs and inadequate follow-up data.

The study was conducted in accordance with the Declaration of Helsinki (as revised in 2013). Ethical approval was obtained from the Medical Ethics Committee of the Seventh Affiliated Hospital of Sun Yat-sen University (No.: KY-2020-024-01). All patient records and information were anonymized and de-identified prior to analysis. Individual consent for this retrospective analysis was waived.

\section{Clinical data collection and processing}

The following variables were extracted directly from our GC database: age, sex, primary tumor site, tumor size, degree of tumor differentiation, preoperative serum carcinoembryonic antigen (CEA) level, Borrmann's classification, type of lymphadenectomy, number of HLNs, number of positive lymph nodes, and follow-up status. Moreover, postoperative pathological $\mathrm{T}$ stage (pT), N stage $(\mathrm{pN})$, and final AJCC TNM stage were recalculated according to the eighth American Joint Committee on Cancer TNM staging system. Patients were divided into four subgroups according to the RML: $0,0-0.1,0.1-0.4$, and $>0.4$, as reported by previous authors (10).

\section{Follow-up and study end points}

All participants were followed every three months in the first two years after hospital discharge, every six months in the subsequent three years, then every year or until death. Follow-up evaluations including: physical examination, serum tumor biomarkers, gastroscopy, and contrastenhanced thorax and abdomen computed tomography (CT). The last follow-up date was December 2019.

The primary end point of this study was OS. OS was defined as the time elapsed between initiation of treatment and death from any cause. OS rates and $95 \%$ confidence intervals (CI) were calculated using the Kaplan-Meier estimator. The log-rank test was used to distinguish differences between the survival curves of different patients' groups. 


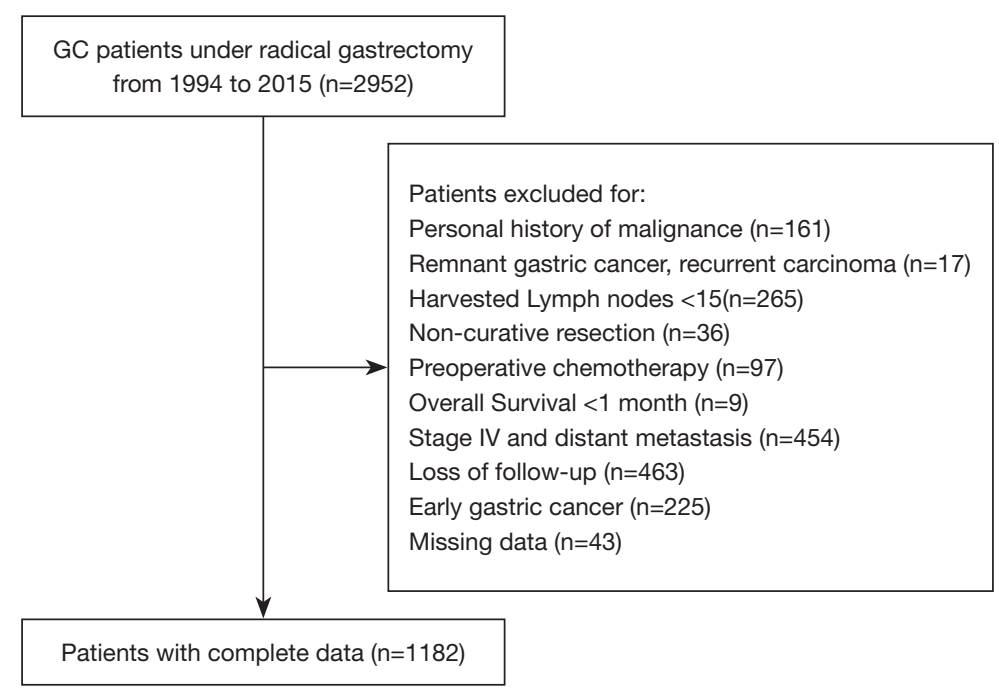

Figure 1 Flowchart describing patient enrollment and exclusion.

\section{Statistical analysis}

Categorical data were reported as absolute numbers (n) with proportion (\%). Groups were compared using chi-square test and Fisher's exact test. Survival rates were analyzed using the Kaplan-Meier survival curves and log-rank test. Univariate analyses were performed with the unadjusted Cox proportional hazards model to calculate the hazard ratio (HR). To identify independent prognostic factors, all significant variables on univariate Cox regression analysis $(\mathrm{P} \leq 0.05)$ were subjected to multivariate Cox regression analysis. Data were analyzed using SPSS (Windows version 22.0; Chicago, IL, USA).

The following variables were analyzed: (I) sex (male or female); (II) age ( $<65$ or $\geq 65$ years); (III) CEA level ( $\leq 5$ or $>5 \mathrm{ng} / \mathrm{mL}$ ); (IV) tumor size $(<5$ or $\geq 5 \mathrm{~cm})$; (V) primary tumor site (lower third, middle third, upper third, or whole stomach); (VI) depth of primary tumor invasion (AJCC) (T2, T3, or T4); (VII) the number of positive lymph nodes (AJCC) (N0, N1, N2, or N3); (VIII) TNM stage (n0, n1, n2, or n3, AJCC, 8th ed., 2017); (IX) differentiation(well, moderate or poor); (X) Borrmann classification of primary tumor (I,II,III,IV); (11) RML (0, 0.1-10\%, 10.1-40\%, and $>40 \%$ ); (XI) number of NLNs (0 to 9,10 to 14 , and $\geq 15$ ).

\section{Results}

\section{Patient characteristics}

In all, 1,182 patients were enrolled in our study, the participant flowchart are shown in Figure 1. The overall median follow- up time was 39 months (interquartile range, 20 to 68 months). Table 1 illustrates the demographic and clinical features of these patients. The 5 -year OS rate of the study cohort was $54.4 \%$. Of these 1182 patients, 798 were men. The mean age was 57.65 years (21-86 years). A total of 860 patients (72.8\%) with MLNs were diagnosed through pathologic examination and assessment. The Borrmann classification was distributed among I (3.6\%), II (19.9\%), III (66.0\%), and IV (9.2\%). The primary tumor site was allocated among the upper third stomach (31.5\%), middle third stomach (24.8\%), lower third stomach (38.7\%), and whole stomach (5.1\%). The median size of the primary tumor was $4.5 \mathrm{~cm}$ (range, $0-23 \mathrm{~cm}$ ). The total number of HLNs removed from patients in our study was 41,125 (median 32, range, 15-102), and 33,365 lymph nodes were negative. Baseline characteristics are summarized in Tables 1 and 2.

\section{Prognostic influence of the RML and NLN count in advanced GC after radical gastrectomy}

In the present study, Kaplan-Meier survival analysis among different RML groups showed that the RML was associated with a poor prognosis (5-year survival rate $13.1 \%$ vs. $42.8 \%$ vs. $69.1 \%$ vs. $81.2 \%, \mathrm{P}<0.001$, Figure $2 A$ ). Sixty-eight patients with less than nine NLNs who presented with an RML $>40 \%$ were distinguished. The median OS duration of these patients was fifteen months, and only eight patients were alive at the end of follow-up. Moreover, 96 patients had an NLN count of 10-14. Among these 96 patients, one had an RML of $0.1-10 \%$, with a median OS duration of 
Table 1 Baseline characteristics of RML among patients

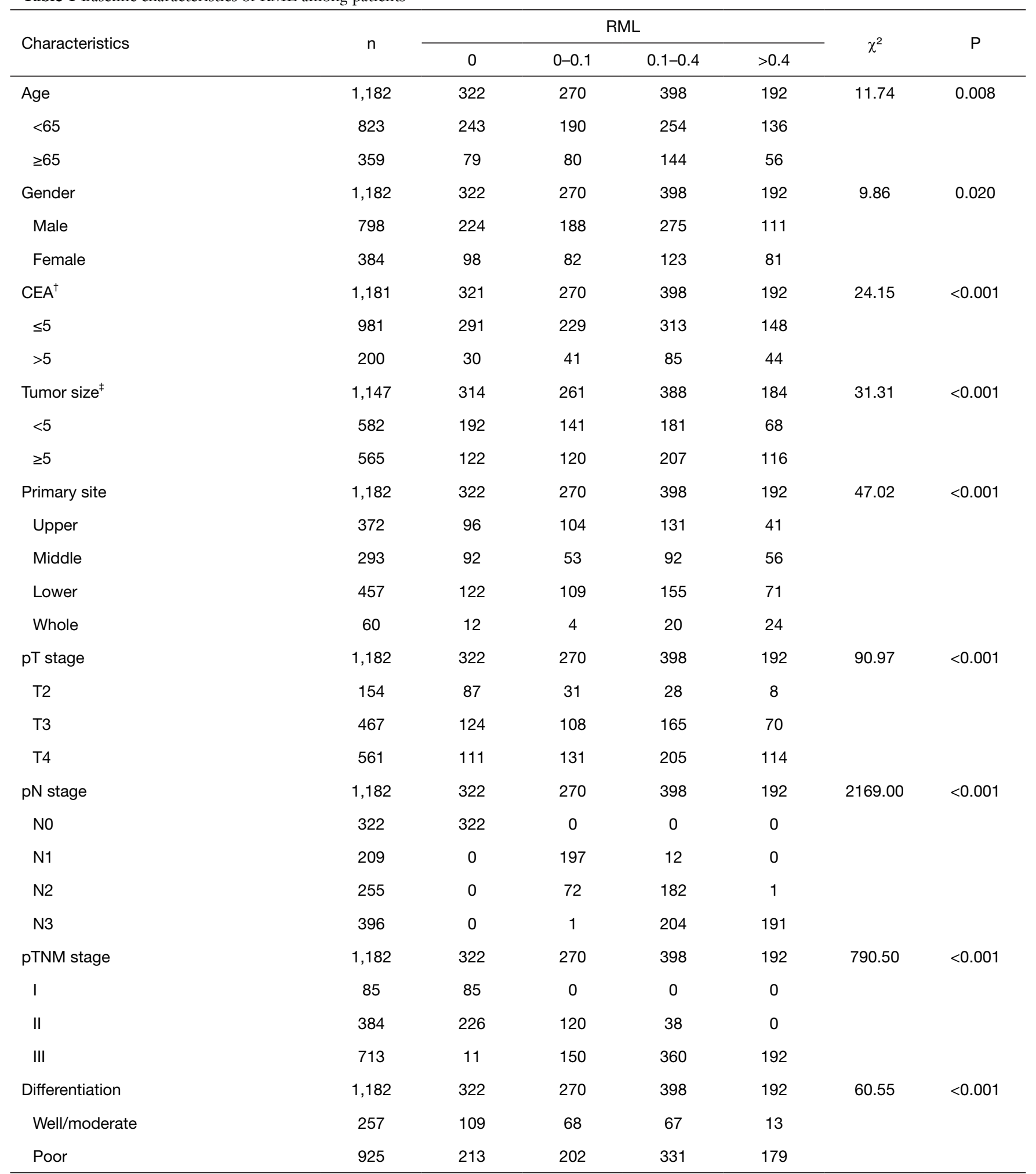

Table 1 (continued) 
Table 1 (continued)

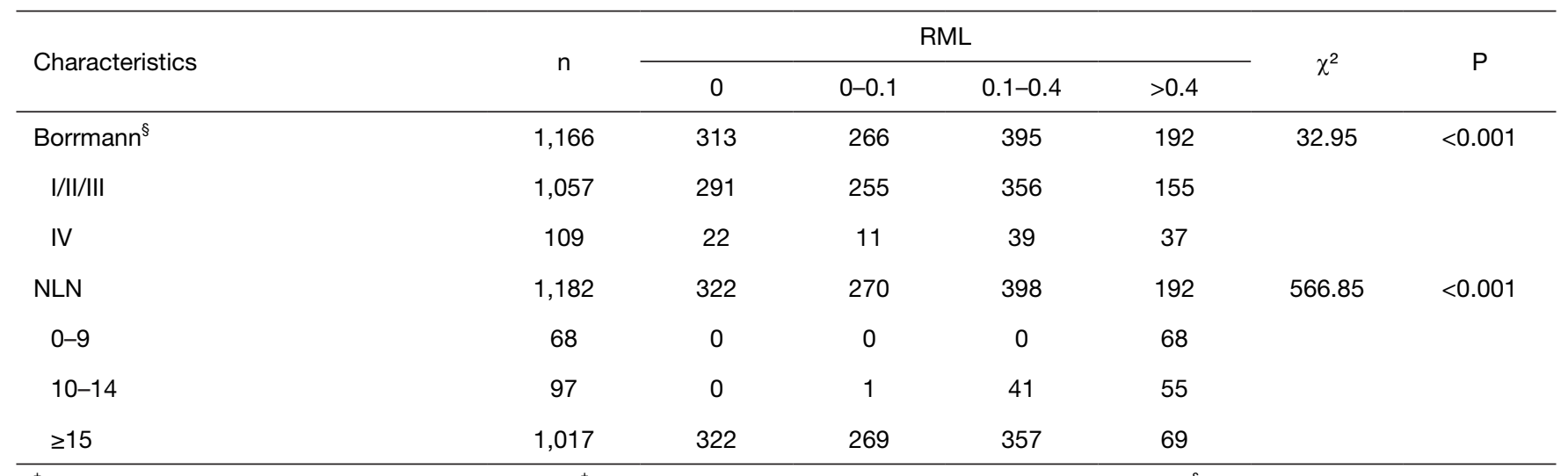

${ }^{\dagger}$, CEA information missing for 1 patient $(0.085 \%) ;{ }^{\ddagger}$, tumor size information missing for 34 patients $(2.8 \%)$; ${ }^{\S}$, Borrmann information missing for 16 patients (1.35\%). CEA, carcinoembryonic antigen; NLN, negative lymph node; RML, ratio between metastatic and harvested lymph nodes.

Table 2 Baseline characteristics of negative lymph node counts among patients

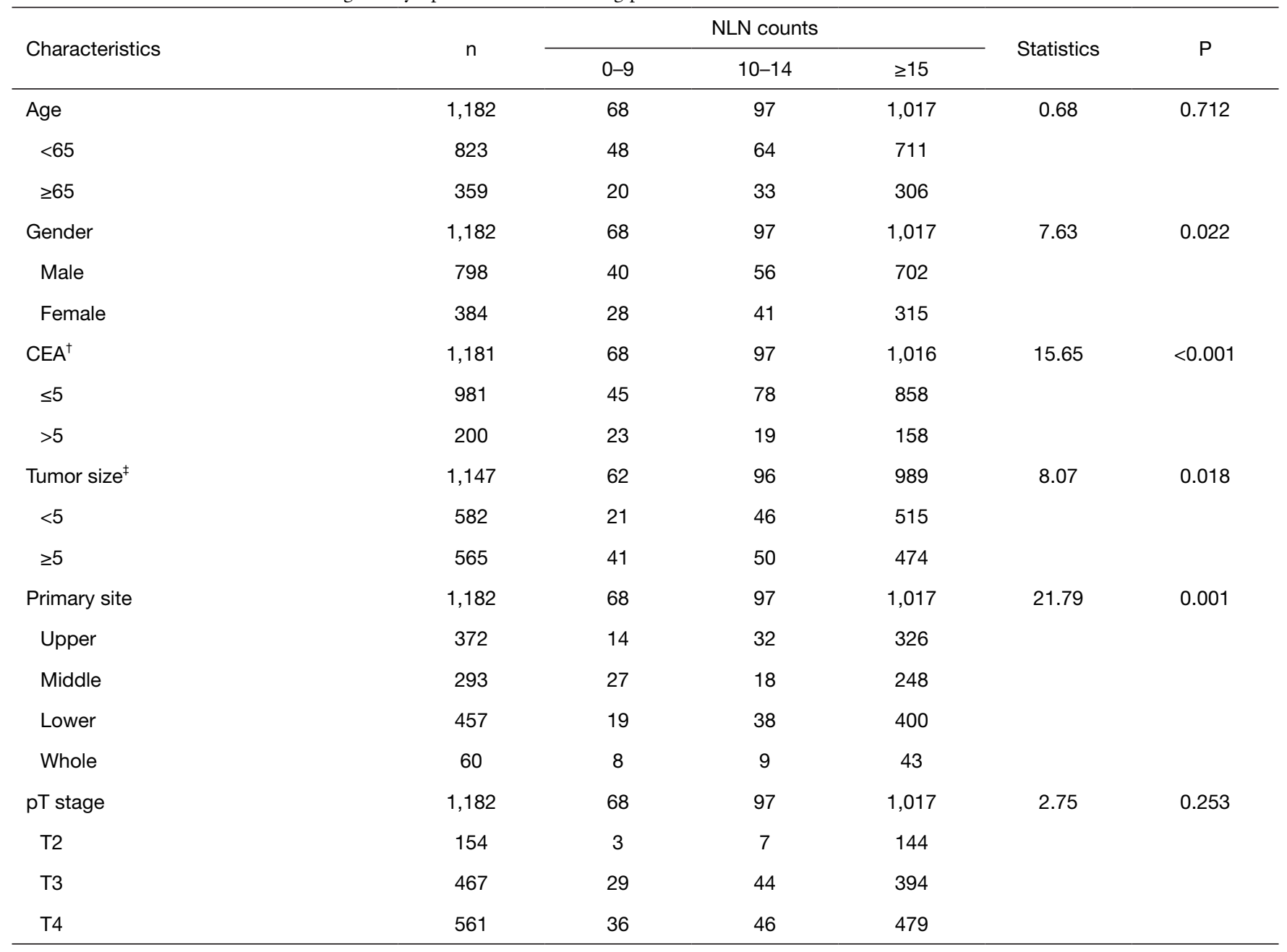

Table 2 (continued) 
Table 2 (continued)

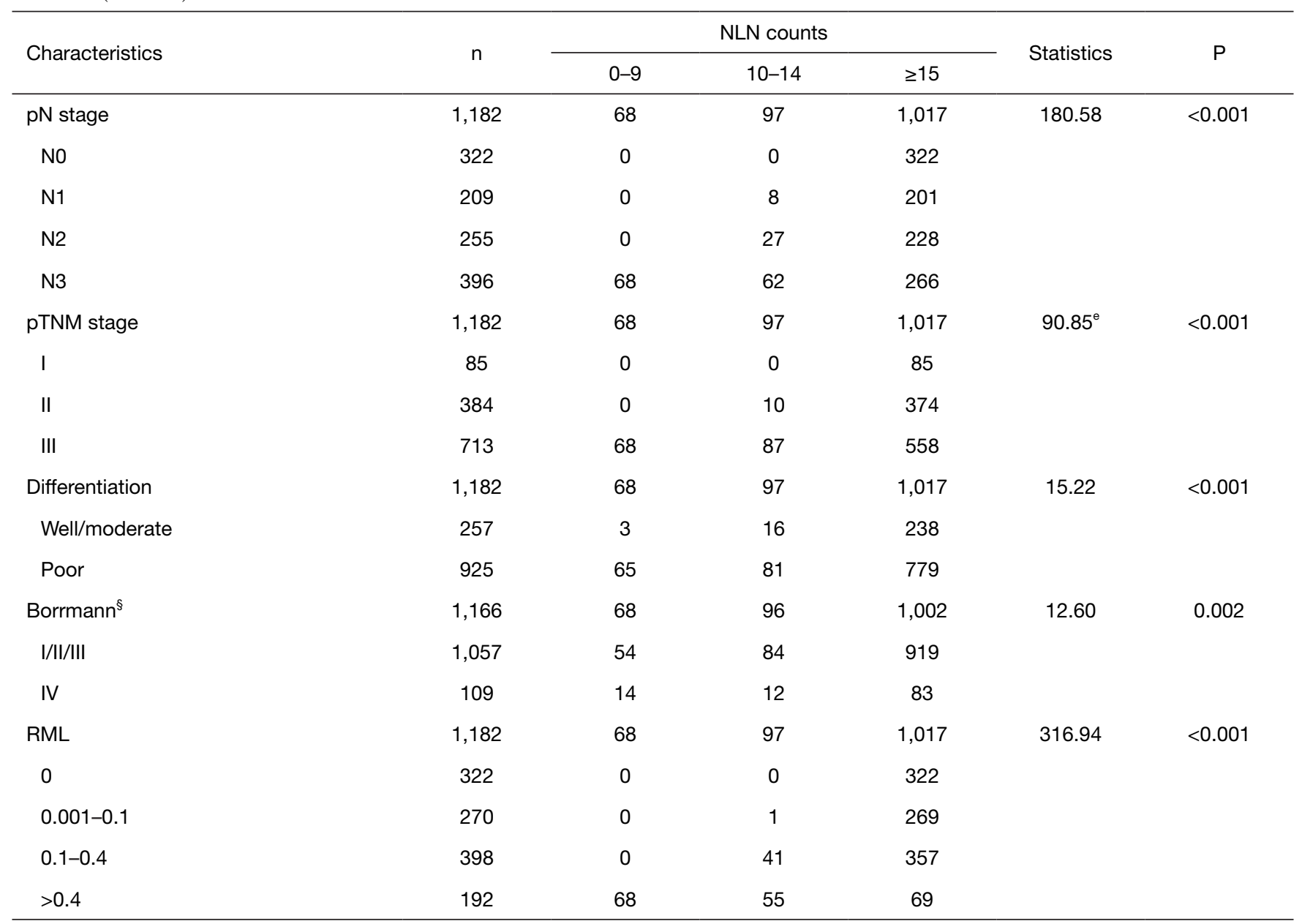

${ }^{\dagger}$, CEA information missing for 1 patient $(0.085 \%) ;{ }^{\ddagger}$, tumor size information missing for 35 patients $(2.8 \%) ;{ }^{\S}$, Borrmann information missing for 16 patients (1.35\%). CEA, carcinoembryonic antigen; NLN, negative lymph node; RML, ratio between metastatic and harvested lymph nodes.

92 months, 41 had an RML of 10.1-40\%, with a median OS duration of 47 months, and 54 had an RML $>40 \%$, with a median OS duration of 19 months. There was a clear trend that the larger the RML was, the shorter the median OS was. Last, 1,017 patients were determined to have $\geq 15$ NLNs. Among these patients, 322 had an RML of 9, with a median OS duration of 142 months, 269 had an RML of $0.1-10 \%$, with a median OS duration of 123 months, 357 had an RML of $10.1-40 \%$, with a median OS duration of 44 months, and 69 had an RML $>40 \%$, with a median OS duration of 18 months. There was a significant correlation between the RML and OS, and the median OS decreased significantly as the RML increased $(\mathrm{P}<0.001)(5$-year survival rate $81.2 \%$ vs. $69.1 \%$ vs. $42.8 \%$ vs. $13.1 \%)$.
After stratification by the AJCC/TNM and $\mathrm{pN}$ stage (Figure $2 \mathrm{~B}-2 \mathrm{H}$ ), we found significant survival differences between patients with stage II disease and an RML of 0 and those with an RML of 10.1-40\% (5-year survival rate $78.3 \%$ vs. $54.7 \%, \mathrm{P}=0.001)$, an RML of $0.1-10 \%$ and an RML of $10.1-40 \%$ (5-year survival rate $72.8 \%$ vs. $54.7 \%, \mathrm{P}=0.019$ ) (Figure 2C) and among patients with stage III disease and an RML of $0.1-10 \%$, an RML of 10.1-40\%, and an RML $>40 \%$ (5-year survival rate $64.8 \%$ vs. 41.5 vs. $13.1 \%, \mathrm{P}<0.001$ ). The median OS showed a decreasing trend with increasing RML (5-year survival rate $36.1 \%$ vs. $13.2 \%, \mathrm{P}<0.001$ ) in stage $\mathrm{pN} 3$ (Figure $2 H$ ). Of note, in the analysis of patients with $\mathrm{pN} 1$ and $\mathrm{pN} 2$ disease, a high RML was not associated with inferior survival $(\mathrm{P}>0.05$, Figure 2F,2G). 

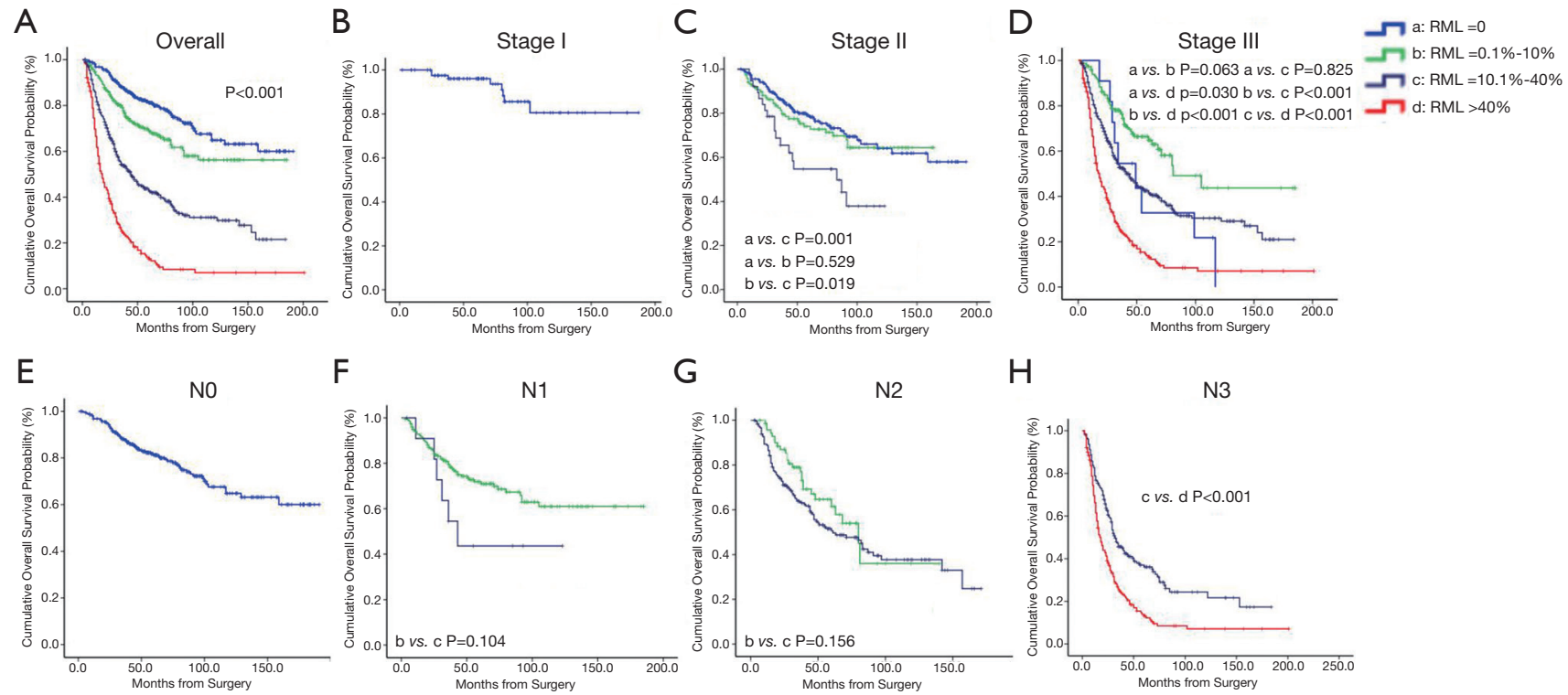

Figure 2 Survival curves of patients according to subgroups: (A) overall; (B) stage I; (C) stage II; (D) stage III; (E) stage pN0; (F) stage pN1; (G) stage $\mathrm{pN} 2 ;(\mathrm{H})$ stage $\mathrm{pN} 3$. In addition, we observed the opposite trend in overall survival after evaluating differences among different NLN groups. The survival analysis revealed that the NLN count was correlated with increased survival rates of gastric cancer patients (5-year survival rate $12.8 \%$ vs. $25.2 \%$ vs. $60.2 \%$, Figure $3 A$, $\mathrm{P}<0.05)$. After stratification by the AJCC/TNM and pN stage (Figure $3 B-3 H)$, we found a significant survival difference between patients with an NLN count $=10-14$ and those with an NLN count $\geq 15$ (5-year survival rate $21.0 \%$ vs. 44.0\%, Figure 3D, $\mathrm{P}<0.001$ ). Remarkably, in our analysis of stage II, pN1 and pN2 gastric cancer patients, the current cutoff of the NLN count was not related with improved survival $(\mathrm{P}>0.05$, Figure $3 C, 3 F, 3 G)$. NLN, negative lymph node; AJCC, American Joint Committee on Cancer; TNM, tumor-node-metastasis.

\section{Univariate and multivariate survival analyses of $O S$}

In the univariate analysis, we found the following 12 clinicopathologic variables to be significantly related to OS: age, sex, CEA level, tumor size, degree of tumor differentiation, primary tumor site, depth of tumor invasion (pT), N stage (pN), AJCC pathological classification (pTNM classification), Borrmann's classification, NLN count, and RML (Table 3). pN stage was excluded from the multivariate analysis due to multicollinearity, and the remaining 11 variables were included in the multivariate Cox proportional hazards model (entry model) to adjust for the influence of covariates.

Five factors that were independently related with OS were revealed through the multivariate analysis: age at surgery $(\mathrm{HR}=1.403, \mathrm{P}<0.001)$, primary tumor site $(\mathrm{P}=0.019)$, pT stage $(\mathrm{P}=0.024)$, Borrmann's classification $(\mathrm{HR}=1.584$, $\mathrm{P}=0.001)$, and $\mathrm{RML}(\mathrm{P}<0.001)$ (Table 3). Patients with an $\mathrm{RML}=0$ had the highest 5 -year survival rate $(81.2 \%)$, followed by patients with an RML of $0.1-10 \%(69.1 \%)$, $10.1-40 \%(42.8 \%)$, and $>40 \%$ (13.1\%) (Figure $2 A)$.
Regarding $\mathrm{pT}$ stage, the 5-year OS rate was highest in patients with stage T2 disease, followed by those with stage $\mathrm{T} 3$ and T4 disease. The 5 -year OS rate was higher in patients with Borrmann I/II/III than in patients with Borrmann IV, in patients with a tumor size $\leq 5 \mathrm{~cm}$ than in patients with a tumor size $>5 \mathrm{~cm}$, and in patients $<65$ years old than in patients $\geq 65$ years old.

To explore the correlations between the RML and number of MLNs and NLN count, we drew two scatter plots with the RML as a dependent variable. We found that the RML was highly associated with the number of MLNs $(\rho=0.973, \mathrm{P}<0.001$, Figure $5 A)$ and decreased as the NLN count increased $(\rho=-0.513, \mathrm{P}<0.001$, Figure $5 B)$.

\section{Discussion}

In our study, we found that the RML was an independent indicator of prognosis in advanced GC patients undergoing gastrectomy with no less than 15 HLNs. This result suggests that a high RML has prognostic value independent of both traditional clinicopathological factors and the 

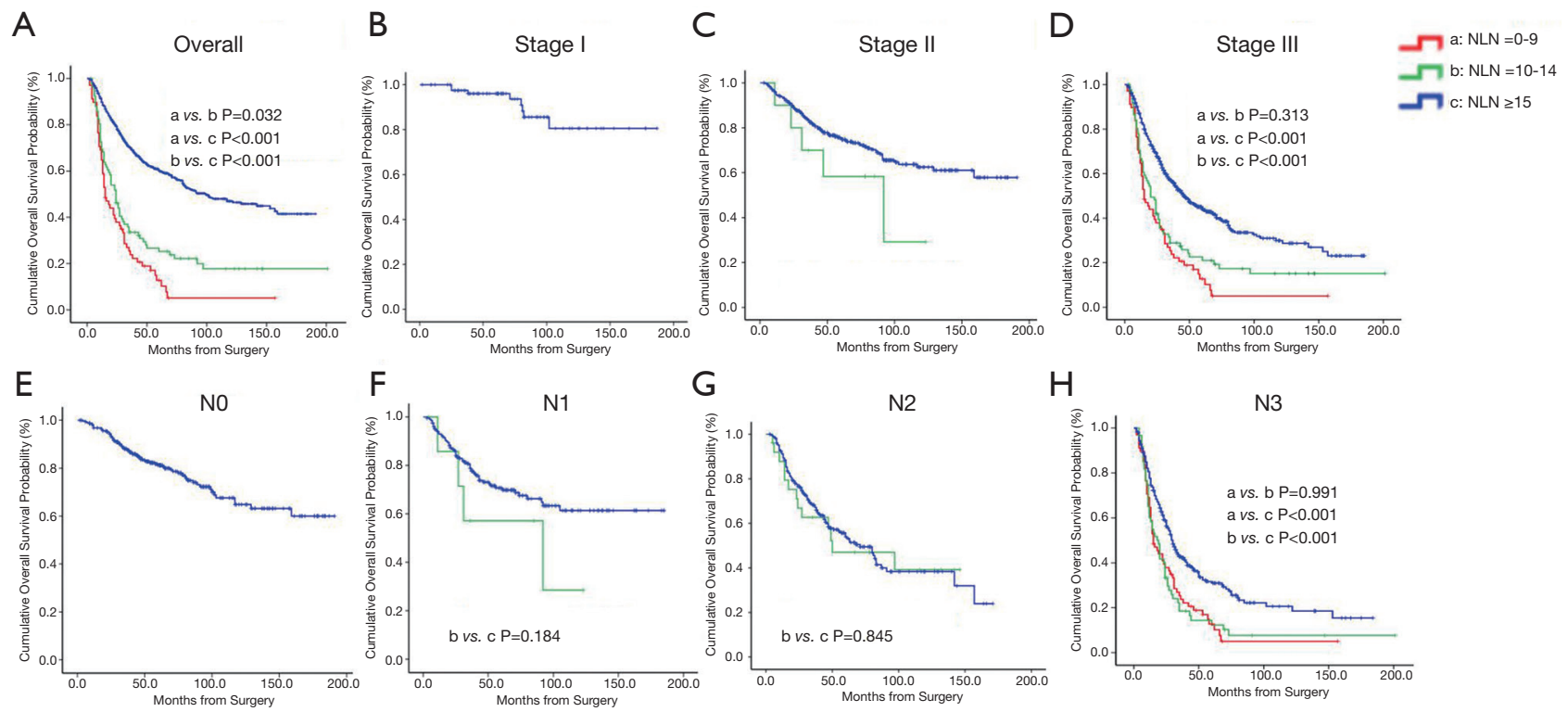

Figure 3 Survival curves of patients according to subgroups: (A) overall; (B) stage I; (C) stage II; (D) stage III; (E) stage pN0; (F) stage pN1; (G) stage $\mathrm{pN} 2 ;(\mathrm{H})$ stage $\mathrm{pN} 3$. Ultimately, our study provides good evidence that the RML can enhance the prognostic prediction when combined with the NLN count (Figure 4A-4C). In the NLN =10-14 group, the RML was associated with inferior survival (5-year survival rate $41.1 \%$ vs. $11.9 \%, \mathrm{P}<0.001$, Figure $4 B$ ). The same result was true among the $\mathrm{NLN} \geq 15$ group $(5$-year survival rate $81.2 \%$ vs. $69.0 \%$ vs. $43.0 \%$ vs. $13.8 \%$, Figure $4 C, \mathrm{P}<0.001)$. RML, the ratio between metastatic and harvested lymph node; NLN, negative lymph node.
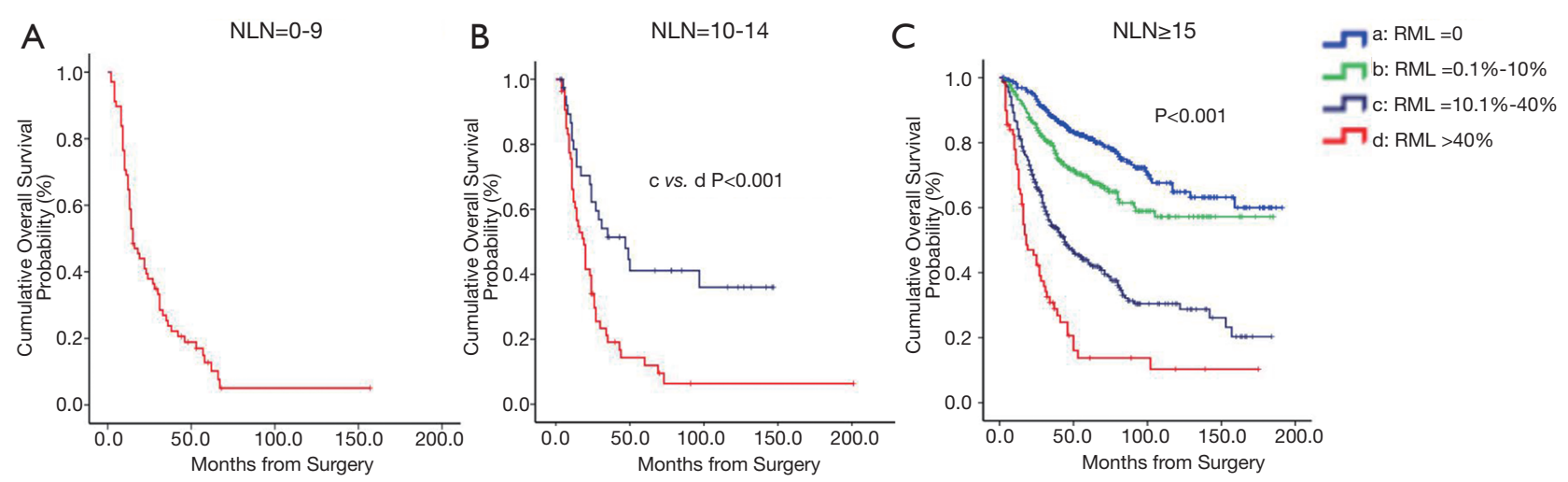

Figure 4 Survival curves of patients according to subgroups: (A) NLN: 0-9; (B) NLN: 10-14; (C) NLN $\geq 15$. NLN, negative lymph node.

extent of lymphadenectomy. We estimated the best cutoff value of the RML to correlate with patient survival. We divided patients into four groups on the basis of the RML and found that a high RML indicated a poor prognosis. The 5-year survival rate was the lowest in the RML $>40 \%$ group, higher in the RML $10.1-40 \%$ and $0.1-10 \%$ groups, and highest in the $\mathrm{RML}=0$ group. Theoretically, a high RML may indicate a poor prognosis (11). Our findings are generally consistent with those from previous studies from both Western and Japanese cohorts despite different cutoff values $(9,12,13)$. Yu et al. (14), in 886 patients who were treated with curative resection, indicated that the RML cutoffs were $0,1-25 \%$, and $>25 \%$, with significant differences among the three groups. In a study by Kodera et al. (15), 656 advanced GC patients who underwent radical gastrectomy with $\mathrm{D} 2$ or higher lymphadenectomy were analyzed, and they chose four cutoffs for the RML: $0 \%, 1-19 \%, 20-60 \%$, and $>60 \%$. Although there is no 
Table 3 Univariate and multivariate analysis for overall survival

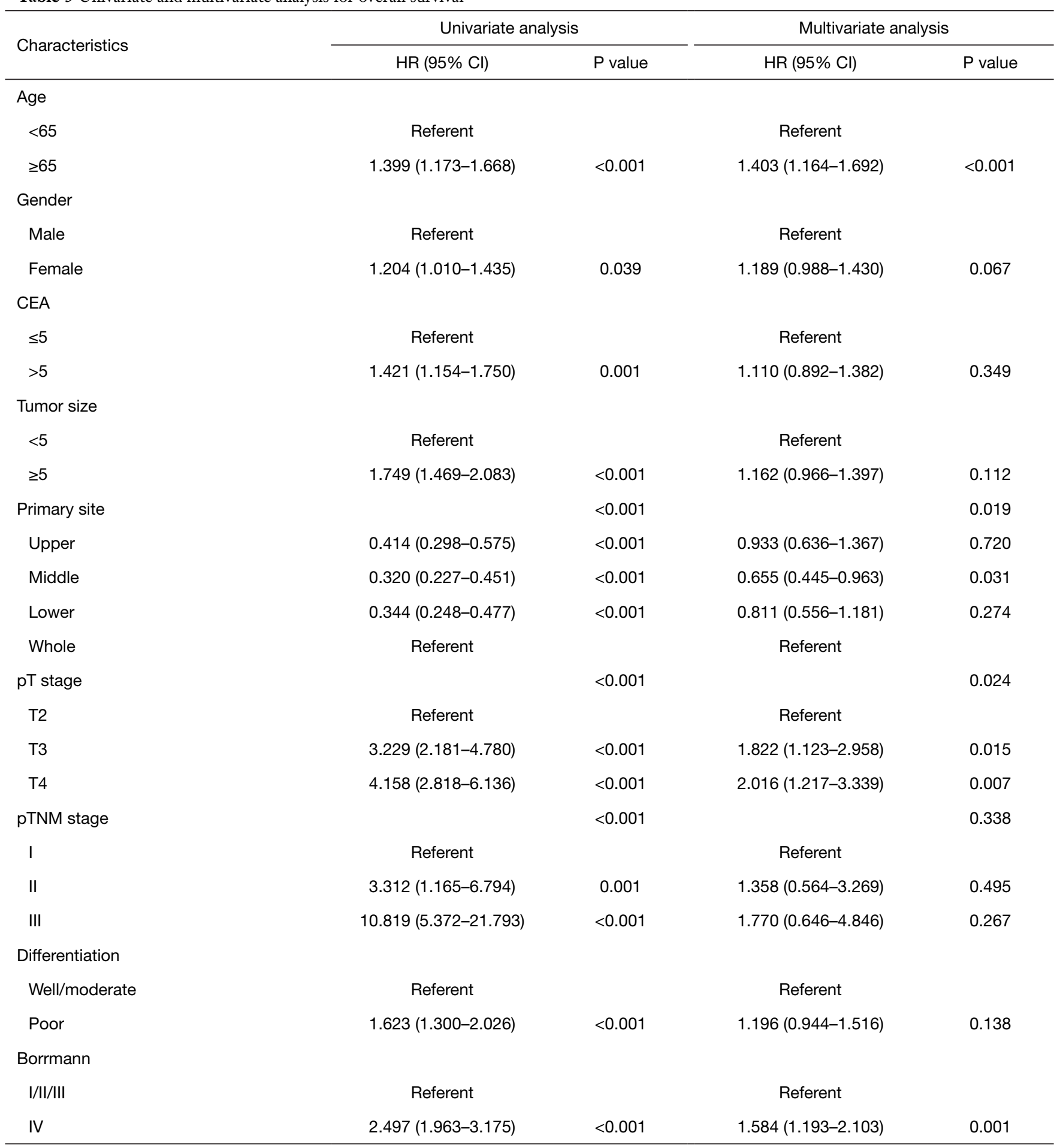

Table 3 (continued) 
Table 3 (continued)

\begin{tabular}{|c|c|c|c|c|}
\hline Characteristics & \multicolumn{2}{|c|}{ Univariate analysis } & \multicolumn{2}{|c|}{ Multivariate analysis } \\
\hline RML & & & & $<0.001$ \\
\hline 0 & Referent & & Referent & \\
\hline $0.001-0.1$ & $1.614(1.178-2.211)$ & 0.003 & $1.268(0.850-1.892)$ & 0.244 \\
\hline$>0.4$ & $8.656(6.529-11.475)$ & $<0.001$ & $4.587(2.780-7.569)$ & $<0.001$ \\
\hline NLN & & $<0.001$ & & 0.747 \\
\hline $0-9$ & Referent & & Referent & \\
\hline $10-14$ & $0.666(0.471-0.941)$ & 0.021 & $1.012(0.697-1.471)$ & 0.948 \\
\hline
\end{tabular}

CEA, carcinoembryonic antigen; NLN, negative lymph node; RML, ratio between metastatic and harvested lymph nodes; HR, hazard ratio; $\mathrm{Cl}$, confidence interval.
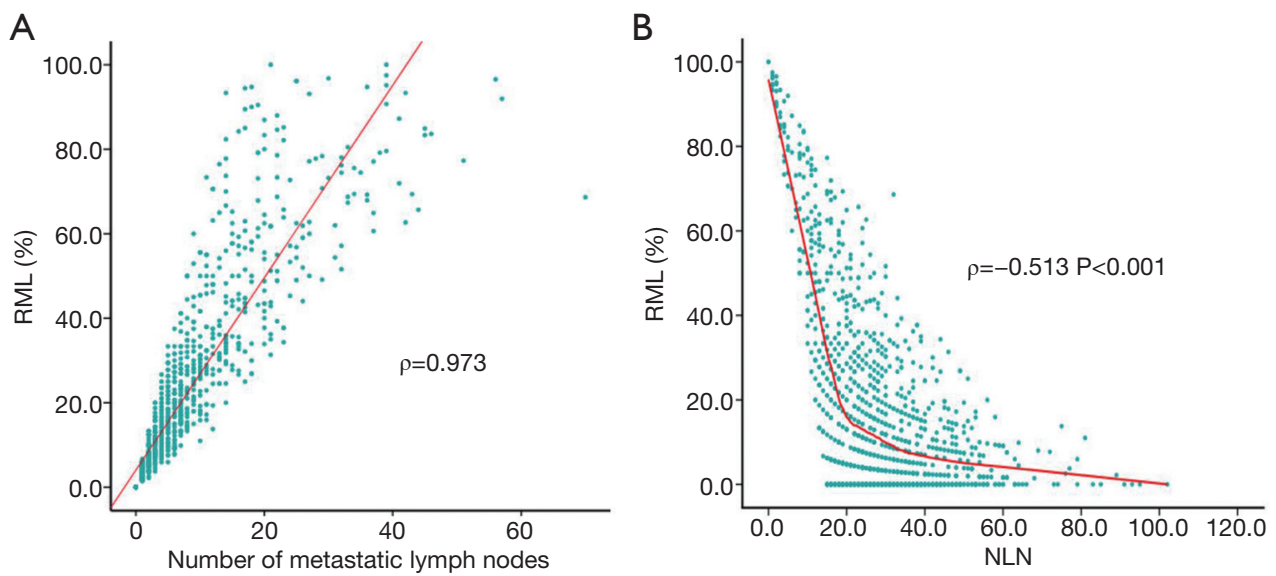

Figure 5 Correlation between RML and number of metastatic lymph nodes (A) and NLN count (B). RML, the ratio between metastatic and harvested lymph node; NLN, negative lymph node.

agreement on the reasonable cutoff values of the RML, all researchers emphasize its clinical significance in terms of simplicity, convenience, reproducibility, and prognostic importance (16).

Moreover, when stratified by the AJCC/TNM stage, we found that the higher the RML was, the worse the prognosis among patients with TNM stage II, III and $\mathrm{pN} 3$ disease. Moreover, when we considered patients in stages $\mathrm{pN} 0, \mathrm{pN} 1$ and $\mathrm{pN} 2$, no statistically significant differences in survival were observed. The findings of our study are generally in accordance with the results of previous studies (9). One possible reason for this is that the RML is distributed mostly near zero. Therefore, the RML is not an effective tool to distinguish GC of the $\mathrm{pN} 0, \mathrm{pN} 1$ and $\mathrm{pN} 2$ stages with less than 6 MLNs.

We divided patients into three groups according to the NLN count and discovered that a higher NLN was related to better survival through Kaplan-Meier survival analysis. Previous studies have reported that so-called NLNs might harbor micrometastasis from the primary tumor (17). In addition, lymph node micrometastasis cannot be diagnosed through a general pathological examination, revealing that the NLN count can be regarded as an important marker and might even be necessary in improving the prognostic 
ability of lymph node status (18). Previous researchers have reported the possibility of recurrence in advanced GC patients without lymph node metastasis even after extended lymphadenectomy (19). Saito et al. (20) suggested that lymph node micrometastasis was significantly related to relapse after radical gastrectomy. Here, we inferred that a higher NLN count means fewer residual tumor cells. However, we found that the NLN count could not serve as an independent factor in advanced GC, as estimated by the multivariate analysis. These results are discrepant with those of Deng (10) and Zhang (21). The possible reasons could be the diverse study population and the inclusion and exclusion criteria. We excluded patients with early GC, those who received preoperative chemotherapy and those with stage IV disease, distant metastasis, and $<15$ HLNs, which is more in line with the actual situation.

The mechanism underlying the correlation between the RML or NLN count and survival has not been determined. There are several possibilities. First, the RML and NLN count may be signs of adequate surgical, pathological, and medical care. Advanced surgeon skills and expertise may be related to more extensive lymph node dissection or a more intensive search for lymph nodes. These factors may contribute to improved outcomes and may reflect institutional endeavors in the therapy of GC patients. Thus, the RML and NLN count may be related to good medical care and may not directly influence the outcome. However, many studies have identified a relationship between the RML or NLN count and outcome, suggesting that when other variables are kept constant, they are still critical (22). Another reason is that an adequate number of lymph nodes rely on surgeons, who vary in attempt and skills as they search for lymph nodes. Many doctors tend to search only for nodes that are visible and palpable (23). Third, according to previous anatomic studies, the number of lymph nodes differs between patients and is affected by disease status (24). The tumor itself may cause lymph nodes to increase in size; germinal centers may also cause the development of lymph nodes, increasing the actual number. If true, the RML and NLN count would indicate potential tumor-host interactions and may have an independent influence on survival (25).

In our creative study, we found a significant relationship between the RML and prognosis in advanced GC patients after gastrectomy with systemic lymphadenectomy, and the RML was identified as an independent predictive indicator of outcome. Stratification analyses by TNM stage and pN stage indicated that both the RML and NLN count could provide better prognostic information for patients with stage III and pN3 GC than those with other stages. Moreover, evaluation of the RML may obviate possible confounding factors associated with the number of lymph nodes excised and the number of gastric lymph nodes, which differ in each individual (26). Unlike the $\mathrm{pN}$ stage classification, the prognostic efficiency was significantly influenced by the number of removed lymph nodes. In summary, our findings emphasize the ability of the RML to distinguish prognostically homogeneous subsets of patients.

However, there were also some limitations to our study. First, the retrospective character of our cohort study is its major limitation. Second, surgical procedures, surgical instruments, surgical skills, examinations of lymph nodes and adjuvant chemotherapies changed during the evolution of the cohort, which may have influenced the findings; such evolution was inherent in long-term follow-up studies. Third, the cohort was comprised of the Chinese population, which may have led to racial bias. However, almost $42 \%$ of GC patients are from China. Therefore, we believe that our present results have representative significance.

In summary, our data suggest that the RML may serve as an independent prognostic indicator in advanced GC patients after radical gastrectomy with standard lymphadenectomy, especially those with stage III and $\mathrm{pN} 3$ disease. Moreover, the RML was highly correlated with the number of MLNs and inversely associated with the NLN count. According to the results presented herein, we suggest utilization of the combination of the RML and NLN count in the prognostic assessment of advanced GC, especially for stage III and pN3 GC patients.

\section{Acknowledgments}

The authors appreciate all supports from Gastrointestinal Surgery Center, First Affiliated Hospital, Sun Yat-sen University, Guangzhou, China.

Funding: This study was supported by grants from Sanming Project of Medicine in Shenzhen (No. SZSM201911010) and the Science and Technology Planning Project of Shenzhen (JCYJ20190809142807444).

\section{Footnote}

Reporting Checklist: The authors have completed the STROBE reporting checklist. Available at https://dx.doi. org/10.21037/jgo-21-212 
Data Sharing Statement: Available at https://dx.doi. org/10.21037/jgo-21-212

Peer Review File: Available at https://dx.doi.org/10.21037/ jgo-21-212

Conflicts of Interest: All authors have completed the ICMJE uniform disclosure form (available at https://dx.doi. org/10.21037/jgo-21-212). The authors have no conflicts of interest to declare.

Ethical Statement: The authors are accountable for all aspects of the work in ensuring that questions related to the accuracy or integrity of any part of the work are appropriately investigated and resolved. The study was conducted in accordance with the Declaration of Helsinki (as revised in 2013). Ethical approval was obtained from the Medical Ethics Committee of the Seventh Affiliated Hospital of Sun Yat-sen University (No.: KY-2020-024-01). All patient records and information were anonymized and de-identified prior to analysis. Individual consent for this retrospective analysis was waived.

Open Access Statement: This is an Open Access article distributed in accordance with the Creative Commons Attribution-NonCommercial-NoDerivs 4.0 International License (CC BY-NC-ND 4.0), which permits the noncommercial replication and distribution of the article with the strict proviso that no changes or edits are made and the original work is properly cited (including links to both the formal publication through the relevant DOI and the license). See: https://creativecommons.org/licenses/by-nc-nd/4.0/.

\section{References}

1. Jemal A, Bray F, Center MM, et al. Global cancer statistics. CA Cancer J Clin 2011;61:69-90.

2. Parkin DM, Bray F, Ferlay J, et al. Global cancer statistics, 2002. CA Cancer J Clin 2005;55:74-108.

3. Abe N, Watanabe T, Suzuki K, et al. Risk factors predictive of lymph node metastasis in depressed early gastric cancer. Am J Surg 2002;183:168-72.

4. Leung WK, Wu MS, Kakugawa Y, et al. Screening for gastric cancer in Asia: current evidence and practice. Lancet Oncol 2008;9:279-87.

5. Kong SH, Lee HJ, Ahn HS, et al. Stage migration effect on survival in gastric cancer surgery with extended lymphadenectomy: the reappraisal of positive lymph node ratio as a proper N-staging. Ann Surg 2012;255:50-8.

6. Yamashita H, Deng J, Liang H, et al. Re-evaluating the prognostic validity of the negative to positive lymph node ratio in node-positive gastric cancer patients. Surgery 2017;161:1588-96.

7. Inoue $\mathrm{K}$, Nakane $\mathrm{Y}$, Iiyama $\mathrm{H}$, et al. The superiority of ratio-based lymph node staging in gastric carcinoma. Ann Surg Oncol 2002;9:27-34.

8. Nitti D, Marchet A, Olivieri M, et al. Ratio between metastatic and examined lymph nodes is an independent prognostic factor after D2 resection for gastric cancer: analysis of a large European monoinstitutional experience. Ann Surg Oncol 2003;10:1077-85.

9. Marchet A, Mocellin S, Ambrosi A, et al. The ratio between metastatic and examined lymph nodes ( $\mathrm{N}$ ratio) is an independent prognostic factor in gastric cancer regardless of the type of lymphadenectomy: results from an Italian multicentric study in 1853 patients. Ann Surg 2007;245:543-52.

10. Deng J, Liang H, Wang D, et al. Enhancement the prediction of postoperative survival in gastric cancer by combining the negative lymph node count with ratio between positive and examined lymph nodes. Ann Surg Oncol 2010;17:1043-51.

11. Bando E, Yonemura Y, Taniguchi K, et al. Outcome of ratio of lymph node metastasis in gastric carcinoma. Ann Surg Oncol 2002;9:775-84.

12. Siewert JR, Böttcher K, Stein HJ, et al. Relevant prognostic factors in gastric cancer: ten-year results of the German Gastric Cancer Study. Ann Surg 1998;228:449-61.

13. Hyung WJ, Noh SH, Yoo CH, et al. Prognostic significance of metastatic lymph node ratio in $\mathrm{T} 3$ gastric cancer. World J Surg 2002;26:323-9.

14. Yu W, Choi GS, Whang I, et al. Comparison of five systems for staging lymph node metastasis in gastric cancer. Br J Surg 1997;84:1305-9.

15. Kodera Y, Yamamura Y, Shimizu Y, et al. Lymph node status assessment for gastric carcinoma: is the number of metastatic lymph nodes really practical as a parameter for $\mathrm{N}$ categories in the TNM Classification? Tumor Node Metastasis. J Surg Oncol 1998;69:15-20.

16. Kunisaki C, Shimada H, Nomura M, et al. Clinical impact of metastatic lymph node ratio in advanced gastric cancer. Anticancer Res 2005;25:1369-75.

17. Huang CM, Lin JX, Zheng CH, et al. Effect of negative lymph node count on survival for gastric cancer after curative distal gastrectomy. Eur J Surg Oncol 
2011;37:481-7.

18. Huang JY, Xu YY, Li M, et al. The prognostic impact of occult lymph node metastasis in node-negative gastric cancer: a systematic review and meta-analysis. Ann Surg Oncol 2013;20:3927-34.

19. Arigami T, Uenosono Y, Yanagita S, et al. Clinical significance of lymph node micrometastasis in gastric cancer. Ann Surg Oncol 2013;20:515-21.

20. Saito H, Osaki T, Murakami D, et al. Recurrence in early gastric cancer--presence of micrometastasis in lymph node of node negative early gastric cancer patient with recurrence. Hepatogastroenterology 2007;54:620-4.

21. Zhang N, Deng J, Wang W, et al. Negative lymph node count as an independent prognostic factor in stage III patients after curative gastrectomy: A retrospective cohort study based on a multicenter database. Int J Surg 2020;74:44-52.

22. Goldstein NS. Lymph node recoveries from 2427

Cite this article as: Jiang J, Chen J, Zhang H, Rao X, Hao T, Li M, Zhang C, Wu W, He Y. Combination of the ratio between metastatic and harvested lymph nodes and negative lymph node count as a prognostic indicator in advanced gastric cancer: a retrospective cohort study. J Gastrointest Oncol 2021;12(5):2022-2034. doi: 10.21037/jgo-21-212
pT3 colorectal resection specimens spanning 45 years: recommendations for a minimum number of recovered lymph nodes based on predictive probabilities. Am J Surg Pathol 2002;26:179-89.

23. Wong SL, Ji H, Hollenbeck BK, et al. Hospital lymph node examination rates and survival after resection for colon cancer. JAMA 2007;298:2149-54.

24. Topor B, Acland R, Kolodko V, et al. Mesorectal lymph nodes: their location and distribution within the mesorectum. Dis Colon Rectum 2003;46:779-85.

25. Johnson PM, Porter GA, Ricciardi R, et al. Increasing negative lymph node count is independently associated with improved long-term survival in stage IIIB and IIIC colon cancer. J Clin Oncol 2006;24:3570-5.

26. Wagner PK, Ramaswamy A, Rüschoff J, et al. Lymph node counts in the upper abdomen: anatomical basis for lymphadenectomy in gastric cancer. Br J Surg 1991;78:825-7. 